TRANSACTIONS OF THE

AMERICAN MATHEMATICAL SOCIETY

Volume 352, Number 6, Pages 2643-2657

S 0002-9947(00)02427-2

Article electronically published on March 2, 2000

\title{
HOMOLOGY DECOMPOSITIONS FOR CLASSIFYING SPACES OF COMPACT LIE GROUPS
}

\author{
ALEXEI STROUNINE
}

\begin{abstract}
Let $p$ be a prime number and $G$ be a compact Lie group. A homology decomposition for the classifying space $B G$ is a way of building $B G$ up to $\bmod p$ homology as a homotopy colimit of classifying spaces of subgroups of $G$. In this paper we develop techniques for constructing such homology decompositions. Jackowski, McClure and Oliver (Homotopy classification of self-maps of BG via G-actions, Ann. of Math. 135 (1992), 183-270) construct a homology decomposition of $B G$ by classifying spaces of $p$-stubborn subgroups of $G$. Their decomposition is based on the existence of a finite-dimensional mod $p$ acyclic $G-C W$-complex with restricted set of orbit types. We apply our techniques to give a parallel proof of the $p$-stubborn decomposition of $B G$ which does not use this geometric construction.
\end{abstract}

\section{INTRODUCTION}

Let $p$ be a fixed prime number and $G$ be a compact Lie group. A homology decomposition for the classifying space $B G$ is a mod $p$ homology isomorphism

$$
\operatorname{hocolim}_{\mathcal{D}} F \underset{p}{\stackrel{\sim}{\longrightarrow}} B G
$$

where $\mathcal{D}$ is a small category, $F$ is functor from $\mathcal{D}$ to the category of spaces, and for each object $d$ of $\mathcal{D}, F(d)$ has the homotopy type of $B H$ for some subgroup $H$ of $G$. Jackowski, McClure and Oliver [6] construct a homology decomposition of $B G$ by classifying spaces of $p$-stubborn subgroups of $G$. Their decomposition is based on the existence of a finite-dimensional mod $p$ acyclic $G-C W$-complex with restricted set of orbit types [6,2.14]. In this paper we give a parallel proof of the $p$-stubborn decomposition of $B G$ which does not use [6, 2.14] and has a potential to be extended to $p$-compact groups.

We will call a set $\mathcal{C}$ of closed subgroups of $G$ a collection if it is closed under the process of taking conjugates in $G$. Let $\mathcal{C}$ be a collection. We define the $\mathcal{C}$-orbit category $\mathcal{O}(\mathcal{C})$ whose objects are the $G$-sets $G / H, H \in \mathcal{C}$, and whose morphisms are $G$-maps. We regard $\mathcal{O}(\mathcal{C})$ as a topological category, where the set of objects is discrete and where the morphism sets have compact-open topology. By $J_{\mathcal{O}(\mathcal{C})}$, or simply $J$, we will denote the inclusion functor from $\mathcal{O}(\mathcal{C})$ to the category of $G$-spaces. Composing $J$ with the Borel construction $E G \times_{G}-$ gives a functor

$$
E G \times_{G} J: \mathcal{O}(\mathcal{C}) \rightarrow \text { Spaces }
$$

Received by the editors December 18, 1997.

1991 Mathematics Subject Classification. Primary 55R35; Secondary 55R40. 
whose value $E G \times{ }_{G} G / H$ at an object $G / H$ has the homotopy type of $B H$. Let * denote the one-point space with the trivial action of $G$. The natural maps $E G \times_{G}$ $G / H \rightarrow E G \times_{G} *=B G$ induce a map

$$
\operatorname{hocolim}_{\mathcal{O}(\mathcal{C})} E G \times_{G} J \rightarrow B G .
$$

Here the operator hocolim is the homotopy colimit defined as a special case of the bar construction (Definition 2.2).

Definition 1.1. Call a collection $\mathcal{C}$ of subgroups of $G$ ample if the map (1.1) induces an isomorphism on $\bmod p$ homology.

Since the Borel construction commutes with taking homotopy colimits

$$
E G \times_{G} \operatorname{hocolim}_{\mathcal{O}(\mathcal{C})} J \cong \operatorname{hocolim}_{\mathcal{O}(\mathcal{C})} E G \times_{G} J
$$

to prove that a collection $\mathcal{C}$ is ample, it suffices to show that in the fibration

$$
\operatorname{hocolim}_{\mathcal{O}(\mathcal{C})} J \rightarrow E G \times_{G} \operatorname{hocolim}_{\mathcal{O}(\mathcal{C})} J \rightarrow B G
$$

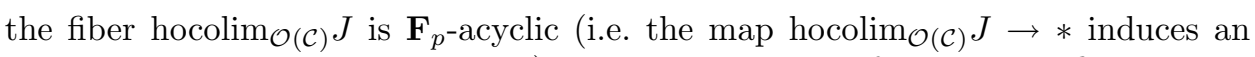
isomorphism on mod $p$ homology). For a collection of subgroups $\mathcal{C}$, we write $E \mathcal{O}(\mathcal{C})=\operatorname{hocolim}_{\mathcal{O}(\mathcal{C})} J$. The action of $G$ on the values of the functor $J$ induces an obvious $G$-action on this space. In section 3 we consider sufficient conditions that imply the $\mathbf{F}_{p}$-acyclicity of $E \mathcal{O}(\mathcal{C})$.

Suppose that the category $\mathcal{O}(\mathcal{C})$, for some collection $\mathcal{C}$, has finite morphism sets. Then the standard Bousfield-Kan cohomology spectral sequence of a homotopy colimit [1 XII.5.8] associated to hocolim ${ }_{\mathcal{O}(\mathcal{C})} E G \times_{G} J$ has the form

$$
E_{2}^{i, j}=\lim _{\mathcal{O}(\mathcal{C})}^{i} H^{j}\left(E G \times_{G} J, \mathbf{F}_{p}\right) \Rightarrow H^{i+j}\left(\operatorname{hocolim}_{\mathcal{O}(\mathcal{C})} E G \times_{G} J, \mathbf{F}_{p}\right) .
$$

Definition 1.2. An ample collection $\mathcal{C}$ of subgroups of $G$ is said to be $\operatorname{sharp}$ if $\mathcal{O}(\mathcal{C})$ is a discrete category (in the sense that the morphism sets of $\mathcal{O}(\mathcal{C})$ have discrete topology) and the spectral sequence (1.2) collapses, that is $E_{2}^{i, j}=0$ for $i>0$ and $E_{2}^{0, j} \approx H^{j}\left(B G, \mathbf{F}_{p}\right)$.

The $E_{2}$-term of the above spectral sequence can be identified with the ordinary equivariant cohomology groups of the $G$-space $E \mathcal{O}(\mathcal{C})$ with certain coefficient systems. In fact, a collection $\mathcal{C}$ is sharp if and only if $E \mathcal{O}(\mathcal{C})$ has equivariant cohomology of a point. In section 4 we obtain a refinement of the equivariant transfer map constructed by Oliver [9] which is used to show that, for suitable collections $\mathcal{C}$, the $E_{2}$-term of the spectral sequence described above has appropriate vanishing properties.

Recall that a compact Lie group $P$ is called $p$-toral if its identity component $P_{0}$ is a torus and $P / P_{0}$ is a finite $p$-group. A $p$-toral subgroup $P$ of $G$ is called $p$-stubborn if $N_{G}(P) / P$ is finite and has no nontrivial normal $p$-subgroups.

The main result of this work is the following.

Theorem 1.3. Suppose that $G$ is a compact Lie group that contains an element of order $p$. Then the collection of nontrivial p-toral subgroups of $G$ is ample and the collection of nontrivial p-stubborn subgroups of $G$ is sharp.

Throughout this paper $p$ is a fixed prime number. The symbol $\mathbf{F}_{p}$ denotes the field with $p$ elements. A map $f: X \rightarrow Y$ between two spaces is said to be an $\mathbf{F}_{p^{-}}$ equivalence or mod $p$ equivalence and is denoted $X \underset{p}{\vec{\longrightarrow}} Y$ if it induces an isomorphism 
$f_{*}: H_{*}\left(X, \mathbf{F}_{p}\right) \rightarrow H_{*}\left(Y, \mathbf{F}_{p}\right)$. A space $X$ is said to be $\mathbf{F}_{p}$-acyclic or $\bmod p$ acyclic if the projection into a one point space $X \rightarrow *$ is an $\mathbf{F}_{p}$-equivalence.

\section{2. $\mathcal{C}$-Resolutions}

Let $\mathcal{C}$ be a collection of subgroups of $G$ and $X$ be a $G$-space. We will call a $G$-map $\varphi: Y \rightarrow X$ a $\mathcal{C}$-resolution of $X$ if the isotropy subgroups of $Y$ are in $\mathcal{C}$ and for each $H$ in $\mathcal{C}$ the map $\varphi$ induces a weak equivalence $\varphi^{H}: Y^{H} \rightarrow X^{H}$ on the fixed point sets. In this section we give a functorial on the category of $G$-space construction of a $\mathcal{C}$-resolution and study its properties.

Throughout this paper a topological category is a category $\mathcal{D}$ with topologized morphism sets such that composition is continuous and for each object $d$ of $\mathcal{D}$ the inclusion $i d_{d} \rightarrow \operatorname{Mor}(d, d)$ is a closed cofibration.

Definition 2.1. Let $\mathcal{D}$ be a small topological category, $\Phi: \mathcal{D}^{o p} \rightarrow$ Top and let $\Psi: \mathcal{D} \rightarrow$ Top be continuous functors. The bar construction $B(\Phi, \mathcal{D}, \Psi)$ is the topological realization $\left|B_{\bullet}\right|$ of the simplicial space $B_{\bullet}$, which in dimension $k$ is the following disjoint union of spaces indexed by ordered sets of $k+1$ objects of $\mathcal{D}$ :

$$
B_{k}=\coprod_{\left(d_{0}, \ldots, d_{k}\right)} \Psi\left(d_{k}\right) \times M o r_{\mathcal{D}}\left(d_{k}, d_{k-1}\right) \times \ldots \times M o r_{\mathcal{D}}\left(d_{1}, d_{0}\right) \times \Phi\left(d_{0}\right) .
$$

The face operators $\delta_{i}$ are induced by composite maps

$$
\operatorname{Mor}_{\mathcal{D}}\left(d_{i+1}, d_{i}\right) \times \operatorname{Mor}_{\mathcal{D}}\left(d_{i}, d_{i-1}\right) \rightarrow \operatorname{Mor}_{\mathcal{D}}\left(d_{i+1}, d_{i-1}\right)
$$

when $0<i<k$, and by evaluation maps

$$
\begin{array}{r}
\operatorname{Mor}_{\mathcal{D}}\left(d_{1}, d_{0}\right) \times \Phi\left(d_{0}\right) \rightarrow \Phi\left(d_{1}\right), \\
\Psi\left(d_{k}\right) \times \operatorname{Mor}_{\mathcal{D}}\left(d_{k}, d_{k-1}\right) \rightarrow \Psi\left(d_{k-1}\right)
\end{array}
$$

when $i$ is respectively 0 and $k$.

The degeneracies are given by appropriate inclusions of identities.

Definition 2.2. Let $*$ denote the constant one point valued functor on $\mathcal{D}$, considered as necessary to be either covariant or contravariant. With the above notation the homotopy colimits of functors $\Phi$ and $\Psi$ are

$$
\operatorname{hocolim} \Phi=B(\Phi, \mathcal{D}, *) ; \operatorname{hocolim} \Psi=B(*, \mathcal{D}, \Psi) .
$$

Recall that the $n$-skeleton $B_{\bullet}^{n}$ of a simplicial space is the smallest subobject that contains $B_{k}$ for all $k \leq n$. The $\bmod p$ homology spectral sequence associated to the filtration

$$
\left|B_{\bullet}^{0}\right| \subset\left|B_{\bullet}^{1}\right| \subset \ldots \subset\left|B_{\bullet}^{n}\right| \subset \ldots
$$

of $\left|B_{\bullet}\right|$ has the $E^{1}$-term $E_{i, j}^{1}=H_{j+i}\left(\left|B_{\bullet}^{i}\right|,\left|B_{\bullet}^{i-1}\right| ; \mathbf{F}_{p}\right) \approx H_{j}\left(B_{i} / s B_{i} ; \mathbf{F}_{p}\right)$ and is first quadrant strongly convergent. Here $s B_{i}$ denotes the subspace of $B_{i}$ which is the union of images of $B_{i-1}$ under the degeneracy operators. A simple application of this spectral sequence is the following:

Lemma 2.3. Suppose that $\mathcal{D}$ is a small topological category, $\Phi, \Phi^{\prime}: \mathcal{D}^{o p} \rightarrow$ Top and $\Psi, \Psi^{\prime}: \mathcal{D} \rightarrow$ Top are continuous functors. If $u: \Phi \rightarrow \Phi^{\prime}$ and $v: \Psi \rightarrow \Psi^{\prime}$ are natural transformations such that for each object $d$ of $\mathcal{D} u(d)$ and $v(d)$ are mod p-equivalences, then the map $B(u, \mathcal{D}, v): B(\Phi, \mathcal{D}, \Psi) \rightarrow B\left(\Phi^{\prime}, \mathcal{D}, \Psi^{\prime}\right)$ is a mod $p$ equivalence. 
Proof. Let $B_{\bullet}$ and $B_{\bullet}^{\prime}$ be the simplicial spaces used to define the bar constructions $B(\Phi, \mathcal{D}, \Psi)$ and $B\left(\Phi^{\prime}, \mathcal{D}, \Psi^{\prime}\right)$, respectively. The transformations $u$ and $v$ induce a map $(u, v): B_{\bullet} \rightarrow B_{\bullet}^{\prime}$ of simplicial spaces such that the corresponding maps $B_{i} \rightarrow B_{i}^{\prime}$ and $s B_{i} \rightarrow s B_{i}^{\prime}$ are mod $p$ homology isomorphisms in each dimension. Since the inclusions $s B_{i} \rightarrow B_{i}$ and $s B_{i}^{\prime} \rightarrow B_{i}^{\prime}$ are cofibrations, $(u, v)$ induces an isomorphism of the spectral sequences described above.

Suppose that $\mathcal{C}$ is a collection of subgroups of $G$ and $X$ is a $G$-space. Define $X_{\mathcal{C}}$, the $\mathcal{C}$ resolution of $X$, to be the bar construction $B\left(\operatorname{Map}_{G}(J, X), \mathcal{O}(\mathcal{C}), J\right)$. There is an obvious $G$-map $\varphi_{\mathcal{C}}(X): X_{\mathcal{C}} \rightarrow X$ induced by the evaluation maps in each dimension of the simplicial space

$$
\begin{aligned}
B_{k}= & \coprod_{\left(G / H_{0}, \ldots, G / H_{k}\right)} G / H_{k} \times \operatorname{Map}_{G}\left(G / H_{k}, G / H_{k-1}\right) \\
& \times \ldots \\
& \times \operatorname{Map}_{G}\left(G / H_{0}, X\right) \rightarrow X .
\end{aligned}
$$

Essentially the space $X_{\mathcal{C}}$ contains all the equivariant homotopy information of $X$ which is detectable by the subgroups of $G$ in $\mathcal{C}$. It is clear from the construction that the isotropy subgroups of $X_{\mathcal{C}}$ are those subgroups $H$ in $\mathcal{C}$ for which the fixed-point space $X^{H}=\operatorname{Map}_{G}(G / H, X)$ is nonempty.

A map $f: X \rightarrow Y$ of $G$-spaces is said to be a weak $G$-equivalence (respectively, a $G$-mod-p-equivalence) if for every subgroup $H$ of $G$ the induced map on the fixed point sets $f^{H}: X^{H} \rightarrow Y^{H}$ is a weak equivalence (respectively, $\bmod p$ equivalence). We will be interested in finding out to what extent the weak $G$-homotopy (homology) type of a $G$ space $X$ is determined by the fixed-point sets $X^{H}$ for some restricted type of subgroups $H$ of $G$. The following facts are simple consequences of general properties of the bar construction.

Theorem 2.4. Let $G$ be a compact Lie group, $\mathcal{C}$ a collection of subgroups of $G$ and $X$ a $G$-space. Then

(a) for every $H$ in $\mathcal{C}$, the map on the fixed-point sets $\left(X_{\mathcal{C}}\right)^{H} \rightarrow X^{H}$ is a homotopy equivalence;

(b) if $H$ is in $\mathcal{C}$, then $\varphi_{\mathcal{C}}(G / H):(G / H)_{\mathcal{C}} \rightarrow G / H$ is a G-equivalence;

(c) if $X$ is a $G-C W$-complex such that for each orbit type $G / H$ of $X, \varphi_{\mathcal{C}}(G / H)$ is a $G$-equivalence, then so is $\varphi_{\mathcal{C}}(X): X_{\mathcal{C}} \rightarrow X$.

Proof. Note that for a topological category $\mathcal{D}$, functors $\Phi: \mathcal{D}^{o p} \rightarrow$ Top and $\Psi$ : $\mathcal{D} \rightarrow$ Top, and an object $d$ of $\mathcal{D}$, the evaluation maps induce natural maps

$$
\begin{aligned}
& B\left(\Phi, \mathcal{D}, \operatorname{Mor}_{\mathcal{D}}(d,-)\right) \stackrel{\sim}{\rightarrow} \Phi(d), \\
& B\left(\operatorname{Mor}_{\mathcal{D}}(-, d), \mathcal{D}, \Psi\right) \stackrel{\sim}{\rightarrow} \Psi(d),
\end{aligned}
$$

which are strong deformation retractions [4, 3.1(5)].

(a) For $H$ in $\mathcal{C}$

$$
\begin{aligned}
\left(X_{\mathcal{C}}\right)^{H} & =B\left(\operatorname{Map}_{G}(J, X), \mathcal{O}(\mathcal{C}), J\right)^{H} \\
& =B\left(\operatorname{Map}_{G}(J, X), \mathcal{O}(\mathcal{C}), \operatorname{Mor}_{\mathcal{O}(\mathcal{C})}(G / H,-)\right) \stackrel{\sim}{\rightarrow} \operatorname{Map}_{G}(G / H, X) \approx X^{H} .
\end{aligned}
$$

(b) For $H$ in $\mathcal{C}$ and $Q$ any subgroup of $G$

$$
\begin{aligned}
\left(G / H_{\mathcal{C}}\right)^{Q} & =B\left(\operatorname{Map}_{G}(J, G / H), \mathcal{O}(\mathcal{C}), J\right)^{Q} \\
& =B\left(\operatorname{Mor}_{\mathcal{O}(\mathcal{C})}(-, G / H), \mathcal{O}(\mathcal{C}), \operatorname{Map}_{G}(G / Q, J)\right. \\
& \sim \\
& \operatorname{Map}_{G}(G / Q, G / H) \approx(G / H)^{Q} .
\end{aligned}
$$


(c) A $G$ - $C W$-complex $X$ is constructed from a collection of " $G$-cells" $\left\{G / H \times D^{n}\right\}$ by taking disjoint unions, pushouts in which one of the maps is a cofibration, and sequential colimits. Clearly, for a subgroup $Q$ of $G$ the fixed point functor $\operatorname{Map}_{G}(G / Q,-)$ preserves this three operations and the bar construction commutes with colimits. Hence $\varphi_{\mathcal{C}}(X)$ is a weak $G$-equivalence if it is one for each $G$-cell of $X$.

Corollary 2.5. If $X$ is a $G$-space and $\mathcal{C}$ contains all isotropy subgroups of $X$, then $\varphi_{\mathcal{C}}(X)$ is a weak $G$-equivalence.

Corollary 2.6. If $f: X \rightarrow Y$ is a $G$-map such that $f^{H}: X^{H} \rightarrow Y^{H}$ is a $\bmod p$ equivalence whenever $H$ is an isotropy subgroup of $X$ or $Y$, then $f$ is a $G$-mod-pequivalence.

Proof. Let $\mathcal{C}$ be a collection of isotropy subgroups of $X$ and $Y$. By $2.4 \varphi_{\mathcal{C}}(X)$ : $X_{\mathcal{C}} \rightarrow X$ and $\varphi_{\mathcal{C}}(Y): Y_{\mathcal{C}} \rightarrow Y$ are weak $G$-equivalences, and by 2.3, $f_{\mathcal{C}}: X_{\mathcal{C}} \rightarrow Y_{\mathcal{C}}$ is a $G$-mod-p-equivalence.

Remark 2.7. Note that the $G$-space $E \mathcal{O}(\mathcal{C})$ associated to a collection $\mathcal{C}$ is the $\mathcal{C}$ resolution of a point $*_{\mathcal{C}}$, so its isotropy subgroups are those in $\mathcal{C}$ and for any $H$ in $\mathcal{C}$ the fixed point set $(E \mathcal{O}(\mathcal{C}))^{H}$ is contractible. We will study the space $E \mathcal{O}(\mathcal{C})$ in more detail in the next section.

We conclude this section by a lemma that considers $\mathcal{C}$ resolutions of transitive $G$-spaces.

Lemma 2.8. Suppose that $H<G$ is a pair of compact Lie groups and $\mathcal{C}$ is a collection of subgroups of $G$. Let $\left.\mathcal{C}\right|_{H}$ be the collection of those subgroups of $H$ which are in $\mathcal{C}$ and $\xi: G \times_{H} E \mathcal{O}\left(\left.\mathcal{C}\right|_{H}\right) \rightarrow G / H$ be the $H$-bundle induced by projection $E \mathcal{O}\left(\left.\mathcal{C}\right|_{H}\right) \rightarrow *$. Then there is a weak $G$-equivalence $f: G \times_{H} E \mathcal{O}\left(\left.\mathcal{C}\right|_{H}\right) \rightarrow G / H_{\mathcal{C}}$ such that $\xi=\varphi_{\mathcal{C}} \circ f$.

Proof. The map $f$ is defined on bar constructions in the following way: First we identify the constant functor $*: \mathcal{O}\left(\left.\mathcal{C}\right|_{H}\right) \rightarrow$ Top with $\operatorname{Map}_{H}(J, *)$ and note that the functor $G \times_{H}$ - commutes with the bar construction, then we obtain a map of bar constructions induced by the functor $G \times_{H}-: \mathcal{O}\left(\left.\mathcal{C}\right|_{H}\right) \rightarrow \mathcal{O}(\mathcal{C})$ :

$$
\begin{aligned}
G \times_{H} B\left(*, \mathcal{O}\left(\left.\mathcal{C}\right|_{H}\right), J\right) & =B\left(\operatorname{Map}_{H}(J, *), \mathcal{O}\left(\left.\mathcal{C}\right|_{H}\right), G \times_{H} J\right) \\
& \rightarrow B\left(\operatorname{Map}_{G}\left(J, G \times_{H} *\right), \mathcal{O}(\mathcal{C}), J\right) .
\end{aligned}
$$

Note that the $G$-spaces $G \times_{H} E \mathcal{O}\left(\left.\mathcal{C}\right|_{H}\right)$ and $G / H_{\mathcal{C}}$ have the same set of isotropy subgroups, namely those subgroups $Q$ in $\mathcal{C}$ which are subconjugates of $H$. For any such $Q$ consider the maps on the fixed point spaces

$$
\xi^{Q}:\left(G \times_{H} E \mathcal{O}\left(\left.\mathcal{C}\right|_{H}\right)\right)^{Q} \stackrel{f^{Q}}{\rightarrow}\left(G / H_{\mathcal{C}}\right)^{Q} \stackrel{\varphi_{\mathcal{C}}^{Q}}{\longrightarrow}(G / H)^{Q} .
$$

The map $\varphi_{\mathcal{C}}^{Q}$ is a weak equivalence by 2.4. The composite $\xi^{Q}$ is a fiber bundle with the fiber over a point $g H$ in $(G / H)^{Q}$ being equal to $\left(E \mathcal{O}\left(\left.\mathcal{C}\right|_{H}\right)\right)^{g^{-1} Q g}$ which is contractible since $\left.g^{-1} Q g \in \mathcal{C}\right|_{H}$. Thus $f$ is a weak equivalence on the fixed point sets for isotropy subgroups of $G \times_{H} E \mathcal{O}\left(\left.\mathcal{C}\right|_{H}\right)$ and $G / H_{\mathcal{C}}$, and hence it is a weak $G$-equivalence by [2.6] 


\section{The $G$-SPACE $E \mathcal{O}(\mathcal{C})$}

As mentioned before, a collection of subgroups $\mathcal{C}$ is ample if the space $E \mathcal{O}(\mathcal{C})$ has $\bmod p$ homology of a point. In this section we state some conditions that imply the mod $p$-acyclicity of this space. We first note that this is a necessary condition for ampleness of $\mathcal{C}$ if $\pi_{0}(G)$ is a $p$-group.

Lemma 3.1. Let $G$ be a compact Lie group such that $\pi_{0}(G)$ is a finite p-group. Then $\mathcal{C}$ is an ample collection of subgroups of $G$ if and only if $E \mathcal{O}(\mathcal{C}) \underset{p}{\sim} *$.

Proof. If $E \mathcal{O}(\mathcal{C}) \underset{p}{\stackrel{\sim}{\longrightarrow}} *$, the conclusion is obvious. Suppose now that $E G \times_{G} E \mathcal{O}(\mathcal{C})$ $\underset{p}{\stackrel{\sim}{B}} B G$. Let $\pi=\pi_{0}(G) \approx G / G_{0}$. Since $\pi$ is a $p$-group it acts nilpotently on $\mathbf{F}_{p}[\pi]$ and the homology isomorphism $E G \times_{G} E \mathcal{O}(\mathcal{C}) \rightarrow E G \times_{G} *$ with coefficients in $\mathbf{F}_{p}$ implies the isomorphism with twisted coefficients

$$
H_{*}\left(E G \times_{G} E \mathcal{O}(\mathcal{C}), \mathbf{F}_{p}\left[G / G_{0}\right]\right) \rightarrow H_{*}\left(E G \times_{G} *, \mathbf{F}_{p}\left[G / G_{0}\right]\right) .
$$

By Shapiro's lemma

$$
H_{*}\left(E G \times_{G} X, \mathbf{F}_{p}\left[G / G_{0}\right]\right) \approx H_{*}\left(E G \times_{G_{0}} X, \mathbf{F}_{p}\right),
$$

thus we have a fibration $E \mathcal{O}(\mathcal{C}) \rightarrow E G \times{ }_{G_{0}} E \mathcal{O}(\mathcal{C}) \rightarrow B G_{0}$ in which the base space is simply-connected and the projection map is a homology isomorphism. Serre spectral sequence on homology of a fibration implies the acyclicity of the fiber.

Definition 3.2. Call a subgroup $H$ in $\mathcal{C}$ a core subgroup of the collection $\mathcal{C}$ if $H$ is normal in $G$ and $H P$ is in $\mathcal{C}$ for every $P$ in $\mathcal{C}$.

The following is a theorem of Quillen in a slightly more general form.

Lemma 3.3. If a collection of subgroups $\mathcal{C}$ has a core subgroup, then $E \mathcal{O}(\mathcal{C})$ is contractible.

Proof. Let $P$ be a core subgroup of $\mathcal{C}$. Consider the following subcollection of $\mathcal{C}$

$$
\mathcal{C}_{P}=\{H \in \mathcal{C}: P \subset H\}
$$

Note that $P$ acts trivially on every object of the associated orbit category $\mathcal{O}\left(\mathcal{C}_{P}\right)$, therefore we can identify the functor $J_{\mathcal{O}\left(\mathcal{C}_{P}\right)}$ with $\operatorname{Mor}_{\mathcal{O}\left(\mathcal{C}_{P}\right)}(G / P,-)$. By (2.1) the space $E \mathcal{O}\left(\mathcal{C}_{P}\right)=B\left(*, \mathcal{O}\left(\mathcal{C}_{P}\right), \operatorname{Mor}_{\mathcal{O}\left(\mathcal{C}_{P}\right)}(G / P,-)\right)$ is contractible.

The map $E \mathcal{O}\left(\mathcal{C}_{P}\right) \rightarrow E \mathcal{O}(\mathcal{C})$, induced by inclusion of categories, is a homotopy equivalence: The inverse is induced by the functor $(-)_{P}: \mathcal{O}(\mathcal{C}) \rightarrow \mathcal{O}\left(\mathcal{C}_{P}\right)$ that sends every object of $G / H$ of $\mathcal{O}(\mathcal{C})$ its $P$-orbit space $(G / H)_{P}$. Since $P$ is a normal subgroup of $G,(G / H)_{P}$ is a transitive $G$ space of type $G / H P$. The projection $G / H \rightarrow(G / H)_{P}$ defines a natural transformation between the identity functor on $\mathcal{O}(\mathcal{C})$ and the composite $\mathcal{O}(\mathcal{C}) \stackrel{(-)_{P}}{\longrightarrow} \mathcal{O}\left(\mathcal{C}_{P}\right) \rightarrow \mathcal{O}(\mathcal{C})$. By [4, 3.1(7)], this transformation gives a homotopy between the maps on $E \mathcal{O}(\mathcal{C})$ induced by these functors.

We denote by $\mathcal{O}_{p}^{+}(G)$ (respectively, $\mathcal{R}_{p}^{+}(G)$ ) the orbit category associated to the collection of all nontrivial $p$-toral subgroups (respectively, all nontrivial $p$-stubborn subgroups).

Remark 3.4. If $G$ has a nontrivial normal $p$-subgroup $P$, then $P$ is a core subgroup of the collection of nontrivial $p$-toral subgroups, hence $E \mathcal{O}_{p}^{+}(G) \simeq *$. This is the original result of Quillen. 
The following theorem will allow us to reduce the proof of the homology decomposition theorem to the case of compact Lie groups with simpler structure (e.g. groups of smaller dimension or groups with $\pi_{0}$ a $p$-group).

Theorem 3.5. Let $G$ be a compact Lie group and $p$ be a prime number. Suppose that there exists a normal subgroup $K$ of $G$ such that for any $p$-toral subgroup $Q$ of $G$ (including the trivial subgroup) $E \mathcal{O}_{p}^{+}(Q K) \underset{p}{\stackrel{\sim}{\vec{p}}} *$. Then $E \mathcal{O}_{p}^{+}(G) \underset{p}{\sim} *$.

Proof. Denote the collection of nontrivial $p$-toral subgroups of $G$ by $\mathcal{C}$. Let $\mathcal{D}=$ $\mathcal{C} \cup\{K Q: Q \in \mathcal{C}\} \cup\{K\}$. Clearly, $\mathcal{D}$ is a collection of subgroups. Consider the following diagram of functors:

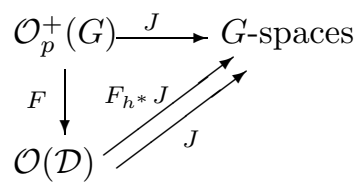

Here $F$ is the inclusion functor and $F_{h^{*}} J$ is the Kan extension of $J$ along $F$ as defined in [4, 5]. Then

$$
E \mathcal{O}_{p}^{+}(G) \cong \operatorname{hocolim}_{\mathcal{O}(\mathcal{D})} F_{h^{*}} \underset{p}{\underset{\sim}{\sim}} \operatorname{hocolim}_{\mathcal{O}(\mathcal{D})} J \cong * .
$$

The first map is an equivalence by the homotopy pushdown theorem [4, 5.5], the third one is the equivalence of Lemma 3.3 (Note that $K$ is a core subgroup of $\mathcal{D}$ ). The map in the middle is constructed in the following way:

For each object $G / H$ of $\mathcal{D}$, by definition the Kan extension is the bar construction $F_{h^{*}} J(G / H)=B\left(M_{o_{\mathcal{D}}}(F, G / H), \mathcal{O}_{p}^{+}(G), J\right)$, which is in fact, the $\mathcal{C}$ resolution of $G / H$. There is a natural transformation $F_{h^{*}} J \rightarrow J$ defined on each object $G / H$ of $\mathcal{D}$ to be the map $\varphi_{\mathcal{C}}(G / H): G / H_{\mathcal{C}} \rightarrow G / H$. We will show that this map is mod $p$ equivalence for each object $G / H$ of $\mathcal{D}$ which will imply the mod $p$ equivalence of the map on the homotopy colimits by 2.3 .

Note that the projection of the fiber bundle $G \times_{H} E \mathcal{O}_{p}^{+}(H) \rightarrow G / H$ is a $\bmod p$ equivalence, since its fiber $E \mathcal{O}_{p}^{+}(H)$ is mod $p$ acyclic by assumption when $H$ is in the form $K Q$ for a $p$-toral $Q$ or it is contractible when $H$ is nontrivial $p$-toral. By 2.8, this projection factors through a weak $G$-equivalence:

$$
G \times_{H} E \mathcal{O}_{p}^{+}(H) \stackrel{\sim}{\rightarrow} G / H_{\mathcal{C}} \stackrel{\varphi \mathcal{C}}{\longrightarrow} G / H .
$$

Therefore, $\varphi_{\mathcal{C}}$ is a mod $p$ equivalence and this finishes the proof.

Remark 3.6. Let $G$ be a positive dimensional compact Lie group, we let $K=G_{0}$, the identity component of $G$. For any $p$-toral $Q, \pi_{0}\left(Q G_{0}\right)$ is a $p$-group, thus Theorem 3.5 combined with Lemma 3.1 allows us to reduce the proof of the homology decomposition theorem to the case of a compact Lie group $\pi_{0}$ which is a $p$-group.

\section{The transfer ON EQUiVARIANT COHOMOLOGY}

Let $G$ be a compact Lie group. We will denote by $\mathcal{O}(G)$ the orbit category $\mathcal{O}(\mathcal{C})$ when $\mathcal{C}$ is the collection of all subgroups of $G$.

Definition 4.1. A coefficient system $M$ for $G$ is a contravariant functor from $\mathcal{O}(G)$ to $A b$, the category of abelian groups, such that if $f$ and $g$ are $G$-homotopic maps in $\mathcal{O}(G), M(f)=M(g)$. 
Let $X$ be a $G$-space and $M$ a coefficient system. By $H_{G}^{*}(X, M)$ we will denote the ordinary equivariant cohomology of $X$ with coefficients in $M$ as defined in [5] and $[12]$.

Suppose $F$ is a functor from a small discrete (in the sense that the set of morphisms has the discrete topology) category $\mathcal{D}$ to the category of $G$-spaces such that $F(d)$ is a transitive $G$-space for each object $d$ of $\mathcal{D}$. There is an equivariant cohomology spectral sequence of $\operatorname{hocolim}_{\mathcal{D}} F$ associated to the skeleton filtration of $\operatorname{hocolim}_{\mathcal{D}} F$ :

$$
E_{2}^{i, j}=\lim _{\mathcal{D}}^{i} H_{G}^{j}(F, M) \Rightarrow H_{G}^{i+j}\left(\operatorname{hocolim}_{\mathcal{D}} F, M\right) .
$$

Note that by the dimension axiom of the ordinary equivariant cohomology $H_{G}^{j}(F, M)=0$ when $j>0$ and $H_{G}^{0}(F, M)=M(F)$. Thus the spectral sequence (4.1) collapses and

$$
H_{G}^{i}\left(\operatorname{hocolim}_{\mathcal{D}} F, M\right) \approx \lim _{\mathcal{D}}^{i} M(F) .
$$

The $\bmod p$ cohomology Bousfield-Kan spectral sequence for hocolim ${ }_{\mathcal{D}} E G \times_{G} F$ has the form

$$
E_{2}^{i, j}=\lim _{\mathcal{D}}^{i} H^{j}\left(E G \times_{G} F, \mathbf{F}_{p}\right) \Rightarrow H^{i+j}\left(\operatorname{hocolim}_{\mathcal{D}} E G \times_{G} F, \mathbf{F}_{p}\right) .
$$

Clearly, its $E_{2}$ term can be identified with (4.2): $M^{j}=H^{j}\left(E G \times_{G}-, \mathbf{F}_{p}\right)$ defines a contravariant coefficient system and $E_{2}^{i, j} \approx H_{G}^{i}\left(\operatorname{hocolim}_{\mathcal{D}} F, M^{j}\right)$. The next proposition is obvious.

Proposition 4.2. Let $G$ be a compact Lie group and $M^{j}=H^{j}\left(E G \times_{G}-, \mathbf{F}_{p}\right)$ a contravariant coefficient system. A collection $\mathcal{C}$ of subgroups of $G$, for which $\mathcal{O}(\mathcal{C})$ is discrete, is sharp if and only if $E \mathcal{O}(\mathcal{C})$ has $M^{j}$ equivariant cohomology of a point

$$
H_{G}^{*}\left(E \mathcal{O}(\mathcal{C}), M^{j}\right) \stackrel{\sim}{\leftarrow} H_{G}^{*}\left(*, M^{j}\right) .
$$

We now describe sufficient conditions that imply the equivariant acyclicity of a $G$-space $X$. First we make a few definitions. Let $P$ be a maximal $p$-toral subgroup of $G$ and $M$ a coefficient system for $G$. Define $\left.M\right|_{P}$, the restriction of $M$ to $P$, to be the composite $\mathcal{O}(P) \stackrel{G \times_{P}-}{\longrightarrow} \mathcal{O}(G) \stackrel{M}{\longrightarrow} A b$. Following the terminology of [7] we will call a subgroup $Q$ of $G$ sub-p-toral if it is contained in a $p$-toral subgroup of $G$. We call a sub- $p$-toral subgroup $Q$ of $G$ p-relevant if it contains an element of order $p$ (equivalently, it is not a finite group of order prime to $p$ ). For a $G$-space $X$ we let $X_{s}=\left\{x \in X: G_{x} \cap P\right.$ is $p$-relevant $\}$, a $P$-invariant subspace of $X$. Recall that a $G$-space $X$ is said to have finitely many orbit types if there are finitely many conjugacy classes of isotropy subgroups of $X$. The following is a result of Slomińska.

Proposition 4.3 ([10, 1.1]). Let $G$ be a Lie group and $P$ be a maximal p-toral subgroup of $G$. Suppose that $X$ is a $G-C W$ complex with finitely many orbit types. With the notation above, if $X_{s}$ is P-mod-p-equivalent to a point, then, for $j>0$,

$$
H_{G}^{*}\left(X, M^{j}\right) \tilde{\sim} H_{G}^{*}\left(*, M^{j}\right) .
$$

Proof. The main idea behind the proof, which can be found in [10], is that the functors $M^{j}$ are restrictions of Mackey functors defined on stable orbit category of $G$ as described in [8], for which a natural equivariant cohomology transfer map is constructed. The transfer exhibits the map $H_{G}^{*}\left(X, M^{j}\right) \sim H_{G}^{*}\left(*, M^{j}\right)$ as a retract of $H_{P}^{*}\left(X,\left.M^{j}\right|_{P}\right) \sim H_{P}^{*}\left(*,\left.M^{j}\right|_{P}\right)$. Since the coefficients $\left.M^{j}\right|_{P}(j>0)$ vanish 
on the $P$-orbits of $X$ outside $X_{s}$, the inclusion map $X_{s} \rightarrow X$ induces an isomorphism $H_{P}^{*}\left(X,\left.M^{j}\right|_{P}\right) \stackrel{\sim}{\rightarrow} H_{P}^{*}\left(X_{s},\left.M^{j}\right|_{P}\right)$. Finally, $H_{P}^{*}\left(X_{s},\left.M^{j}\right|_{P}\right)$ is isomorphic to $H_{P}^{*}\left(*,\left.M^{j}\right|_{P}\right)$ by [6, A.13].

Remark 4.4. The problem one faces trying to get a result analogous to 4.3 when $j=0$ is that the functor $\left.M^{0}\right|_{P}$ does not vanish outside of $X_{s}$ and the map $H_{P}^{*}\left(X,\left.M^{j}\right|_{P}\right) \rightarrow H_{P}^{*}\left(X_{s},\left.M^{j}\right|_{P}\right)$ induced by inclusion is not necessarily an isomorphism. Another result of Slomińska [10 1.2] covers the case $j=0$, however it does depend on the Jackowski-McClure-Oliver construction. We will give an independent proof, which is based on ideas that we are planning to exploit later.

Proposition 4.5. Let $G$ be a compact Lie group, let $P$ be a maximal p-toral subgroup of $G$, and let $X$ be a $G-C W$-complex such that each point in $X$ is fixed by an element in $G$ of order $p$. If $X_{s} / P$ is mod-p-acyclic, then so is the orbit space $X / G$.

Corollary 4.6. Let $G, P$, and $X$ be as in, 4.5. Assume, in addition, that $X$ has finitely many orbit types. If $X_{s}$ is $P$-mod-p-equivalent to a point, then

$$
H_{G}^{*}\left(X, M^{0}\right) \sim H_{G}^{*}\left(*, M^{0}\right) .
$$

Proof. Note that the coefficient system $M^{0}$ is a constant system,

$$
M^{0}(G / H) \approx H^{0}\left(B H, \mathbf{F}_{p}\right)=\mathbf{F}_{p}
$$

and the equivariant cohomology $H_{G}^{*}\left(X, \mathbf{F}_{p}\right)$ is the ordinary cohomology of the orbit space $H^{*}\left(X / G, \mathbf{F}_{p}\right)$. By [6, A.13] $H_{P}^{*}\left(X_{s},\left.M^{0}\right|_{P}\right)$ is isomorphic to $H_{P}^{*}\left(*,\left.M^{0}\right|_{P}\right)$. The result follows from 4.5 .

Our proof of 4.5 is analogous to the proof of 4.3 and is based on the construction of the transfer map given by Oliver 9]. For the rest of this section $H^{*}$ denotes the Čech cohomology.

Theorem $4.7([9])$. Let $G$ be a compact Lie group, $P$ a subgroup of $G$ and $R$ any coefficient group, then for any paracompact and perfectly normal $G$-space $X$, there is a transfer homomorphism

$$
\operatorname{trf}: H^{*}(X / P ; R) \rightarrow H^{*}(X / G ; R),
$$

natural in $X$, and such that the composite

$$
\operatorname{tr} f \circ \pi^{*}: H^{*}(X / G ; R) \rightarrow H^{*}(X / G ; R),
$$

where $\pi$ denotes the projection $X / P \rightarrow X / G$, is multiplication by the Euler characteristic $\chi(G / P)$.

Note that any $G-C W$-complex is paracompact and perfectly normal. We will show the following:

Lemma 4.8. If $P$ is a maximal p-toral subgroup of $G, X$ is a $G$-space such that each point in $X$ is fixed by an element in $G$ of order $p$, and $R=\mathbf{F}_{p}$, then the transfer map (4.3) factors as $H^{*}\left(X / P ; \mathbf{F}_{p}\right) \stackrel{i^{*}}{\rightarrow} H^{*}\left(X_{s} / P ; \mathbf{F}_{p}\right) \rightarrow H^{*}\left(X / G ; \mathbf{F}_{p}\right)$, where $i^{*}$ is induced by inclusion $X_{s} \rightarrow X$.

Before proving 4.8 we recall basic properties of the Dold fixed point index [2] and the key constructions from [9]:

The fixed point index assigns an integer $\operatorname{Ind}(f)$ to any map $f: U \rightarrow M$, where $M$ is an $E N R$ (Euclidean neighborhood retract), $U$ an open subset of $M$, and 
Fix $(f)=\{u \in U: f(u)=u\}$ is compact. We will use the following properties of the index:

Lemma 4.9 ([2, 1.3-1.8]). Let $f: U \rightarrow M$ be as above.

(a) If $V \subseteq U$ is open and $F i x(f) \subseteq V$, then $\operatorname{Ind}\left(\left.f\right|_{V}\right)=\operatorname{Ind}(f)$.

(b) If $U=\coprod_{i=0}^{n} U_{i}$, a finite disjoint union of open sets, then $\operatorname{Ind}(f)=\sum_{i=0}^{n} \operatorname{Ind}\left(\left.f\right|_{U_{i}}\right)$.

(c) If $\varphi: U \times I \rightarrow M$ is a homotopy such that Fix $(\varphi)=\{u \in U: \varphi(u, t)=u$ for some $t \in I\}$ is compact, then $\operatorname{Ind}\left(\varphi_{0}\right)=\operatorname{Ind}\left(\varphi_{1}\right)\left(\varphi_{t}=\varphi(-, t), t \in I\right)$.

(d) If $M$ and $M^{\prime}$ are ENR's, $U \subseteq M$ and $U^{\prime} \subseteq M^{\prime}$ are open sets, and $f$ : $U \rightarrow M^{\prime}, g: U^{\prime} \rightarrow M$ are maps, then the two composites $g f: f^{-1}\left(U^{\prime}\right) \rightarrow M$ and $f g: g^{-1}(U) \rightarrow M^{\prime}$ have homeomorphic fixed point sets and, if these sets are compact, $\operatorname{Ind}(g f)=\operatorname{Ind}(f g)$.

As a simple consequence of 4.9 we have the following:

Lemma 4.10. Suppose that $M$ is an ENR, $V$ an open subset of $M, f: V \rightarrow M$ is a compactly fixed map and $q: M \rightarrow M$ is an automorphism of $M$. Then the map $q f q^{-1}: q(V) \stackrel{q^{-1}}{\rightarrow} V \stackrel{f}{\rightarrow} M \stackrel{q}{\rightarrow} M$ has the same index as $f$.

Proof. Note that $q f: V \rightarrow M$ and $\left.q^{-1}\right|_{q(V)}: q(V) \rightarrow M$ are maps whose two composites have the same index by $4.9(\mathrm{~d})$.

Definition 4.11. Let $X$ be a space and $Y$ be a $G$-space. A homotopy

$$
\varphi: X \times I \rightarrow Y / G
$$

is called stratum decreasing if for any $x$ in $X$ and $t \geq s$ in $I$ the isotropy subgroups of the orbit $\varphi(x, t)$ in $Y$ are subconjugates to the ones of $\varphi(x, s)$.

Lemma 4.12 (9, Theorem 2]). Let $G$ be a compact Lie group acting on spaces $X$ and $Y$, where $X$ is paracompact and perfectly normal and $Y$ is completely regular. Then, for any $G$-map $f: X \rightarrow Y$, any stratum decreasing homotopy of $f / G$ can be lifted to a $G$-homotopy of $f$.

Lemma 4.13 (9, Theorem 3]). For any pair $P \subset G$ of compact Lie groups and any paracompact perfectly normal $G$-space $X$ there is a stratum decreasing homotopy $\varphi: X / P \times I \rightarrow X / P$ of the identity on $X / P$, commuting with the projection $\pi: X / P \rightarrow X / G$ and such that the restriction of $\pi:$

$$
\hat{\pi}: \operatorname{Fix}\left(\varphi_{1}\right) \rightarrow X / G\left(\varphi_{1}=\varphi(-, 1)\right)
$$

has zero-dimensional fibers.

The "stratum decreasing" property of $\varphi$ implies that it restricts to a homotopy $\varphi_{s}: X_{s} / P \times I \rightarrow X_{s} / P$ with analogous properties. We will denote by $\hat{\pi}_{s}$ the restriction of $\hat{\pi}$ to Fix $_{s}\left(\varphi_{1}\right)=\operatorname{Fix}\left(\varphi_{1}\right) \cap X_{s} / P$.

Let $\mathcal{H}$ (respectively, $\mathcal{H}_{s}$ ) denote the sheaf over $X / G$ induced by the presheaf $U \rightarrow H^{0}\left(\hat{\pi}^{-1}(U) ; \mathbf{F}_{p}\right)$ (respectively, $\left.U \rightarrow H^{0}\left(\hat{\pi}_{s}^{-1}(U) ; \mathbf{F}_{p}\right)\right)(U$ open in $X / G)$. Since $\hat{\pi}$ and $\hat{\pi}_{s}$ are closed maps, $\mathcal{H}$ (respectively, $\left.\mathcal{H}_{s}\right)$ has stalks $\mathcal{H}_{x}=H^{0}\left(\hat{\pi}^{-1}(x) ; \mathbf{F}_{p}\right)$ (respectively, $\left.\left(\mathcal{H}_{s}\right)_{x}=H^{0}\left(\hat{\pi}_{s}^{-1}(x) ; \mathbf{F}_{p}\right)\right)(x \in X / G)$. Since each of the sets $\hat{\pi}^{-1}(x)$, $\hat{\pi}_{s}^{-1}(x)$ is zero-dimensional,

$$
H^{*}\left(F i x\left(\varphi_{1}\right), \mathbf{F}_{p}\right) \cong H^{*}(X / G, \mathcal{H}) \text { and } H^{*}\left(F_{i x}\left(\varphi_{1}\right), \mathbf{F}_{p}\right) \cong H^{*}\left(X / G, \mathcal{H}_{s}\right) .
$$


For any $x \in X / G$, choose $\tilde{x}$ in the orbit $x$. Let $K$ denote the isotropy subgroup $G_{\tilde{x}}$ of $\tilde{x}$. The fiber $\pi^{-1}(x)$ can be identified with the orbit space $K \backslash G / P$ of the $K$ action on $G / P$ through the homeomorphism $K \backslash G / P \rightarrow \pi^{-1}(x) \subseteq X / P$ that sends the equivalence class $[g P]$ in $K \backslash G / P$ to $\left[g^{-1} \tilde{x}\right]$ in $X / P$. Under this identification $\varphi$ restricts to a homotopy $\left.\varphi\right|_{K \backslash G / P \times I}: K \backslash G / P \times I \rightarrow K \backslash G / P$, which is stratum decreasing with respect to the action of $K$ by $[9$ Lemma 5 (iii)]. By 4.12 it can be lifted to a $K$-equivariant homotopy $\psi$ of the identity map on $G / P$. Note that the fixed point set of the resulting map $\psi_{1}=\psi(-, 1)$ projects into $\hat{\pi}^{-1}(x) \subset K \backslash G / P$. For any set $Y \subset \hat{\pi}^{-1}(x) \subset K \backslash G / P$ which is both open and closed in $\hat{\pi}^{-1}(x)$ let $\bar{U}$ be an open set of $Y$ in $K \backslash G / P$ that separates $Y$ from the rest of $\hat{\pi}^{-1}(x)$ and let $U$ be the preimage of $\bar{U}$ in $G / P$. Let $I(Y)$ denote the Dold fixed point index of $\left.\psi_{1}\right|_{U}: U \rightarrow G / P$. This index is independent of the choice of $\tilde{x}, \psi$ and $U$. For any space $X$, the cohomology group $H^{0}\left(X, \mathbf{F}_{p}\right)$ can be identified with $C\left(X, \mathbf{F}_{p}\right)$, the group of continuous functions from $X$ to $\mathbf{F}_{p}$ (with the discrete topology). Oliver defines a map $J(\varphi)$ from the sheaf $\mathcal{H}$ to the constant sheaf $X / G \times \mathbf{F}_{p}$, which is a homomorphism $J(\varphi)_{x}: H^{0}\left(\hat{\pi}^{-1}(x) ; \mathbf{F}_{p}\right) \cong C\left(\hat{\pi}^{-1}(x) ; \mathbf{F}_{p}\right) \rightarrow \mathbf{F}_{p}$ on stalks that send $u \in C\left(\hat{\pi}^{-1}(x) ; \mathbf{F}_{p}\right)$ to $\sum_{r \in \mathbf{F}_{p}} r \cdot I\left(u^{-1}(r)\right)$. The map $J(\varphi)$ is continuous [9] 14], and hence a sheaf homomorphism.

Our proof of 4.8 is based on the following fact:

Lemma 4.14. With the notation above, $I(Y)$ is divisible by $p$ for every $Y \subset$ $\hat{\pi}^{-1}(x)-\hat{\pi}_{s}^{-1}(x)$ which is both open and closed in $\hat{\pi}^{-1}(x)$.

Proof. Let $\bar{U}$ be a neighborhood of $Y$ in $K \backslash G / P \cong \pi^{-1}(x)$ that separates $Y$ from $\left(X_{s} \cap \pi^{-1}(x)\right) \cup\left(\hat{\pi}^{-1}(x)-Y\right)$ and let $U$ be its preimage in $G / P$. It follows from the definition of $X_{s}$ that the $K$-isotropy subgroups of points of $U$ are finite of order prime to $p$. Let $Q$ be a cyclic subgroup of $K$ of order $p$. Clearly, it acts freely on $U$. Consider projections $U \stackrel{\alpha}{\rightarrow} U / Q \stackrel{\beta}{\rightarrow} U / K$. Since any subspace of a paracompact and perfectly normal space is paracompact and perfectly normal ([9 Lemma 1 (2)]), by 4.13 there exists a stratum decreasing homotopy of the identity $\omega: U / Q \times I \rightarrow U / Q$ which commutes with the projection $U / Q \rightarrow U / K$, and such that the resulting map $\omega_{1}$ has a zero-dimensional fixed-point set on each fiber of $U / Q \rightarrow U / K$. Composing $\omega$ with $\psi_{1} / Q$ we get a homotopy

$$
U / Q \times I \stackrel{\omega}{\rightarrow} U / Q \stackrel{\psi_{1} / Q}{\rightarrow}(G / P)_{Q}
$$

which, by 4.12, can be lifted to a $Q$-equivariant homotopy $\bar{\omega}: U \times I \rightarrow G / P$ of $\left.\psi_{1}\right|_{U}$. Note that the fixed point set of $\bar{\omega}$ projects into the fixed point set $Y$ of $\left.\varphi_{1}\right|_{U / K}$ : $U / K \rightarrow K \backslash G / P$, therefore Fix $(\bar{\omega})$ is compact. By 4.9 (c) $\operatorname{Ind}\left(\left.\psi_{1}\right|_{U}\right)=\operatorname{Ind}\left(\bar{\omega}_{1}\right)$. Now the $p$-fold covering $\alpha$ projects $F i x\left(\bar{\omega}_{1}\right)$ into a compact zero-dimensional space $F i x\left(\bar{\omega}_{1} / Q\right)=F i x\left(\omega_{1}\right) \cap \beta^{-1}(Y)$. Choose a finite covering $\left\{U_{i}\right\}_{i=0, \ldots, m}$ of $F i x\left(\bar{\omega}_{1} / Q\right)$ by open subsets of $U / Q$ which are evenly covered by $\alpha$. Since Fix $\left(\bar{\omega}_{1} / Q\right)$ is zerodimensional, the open covering $\left\{U_{i} \cap\right.$ Fix $\left.\left(\bar{\omega}_{1} / Q\right)\right\}$ of Fix $\left(\bar{\omega}_{1} / Q\right)$ has a refinement $\left\{K_{j}\right\}_{j=0, \ldots, k}$ such that $K_{j}$ 's are disjoint. Since $U / Q$ is normal and the set $\left\{K_{j}\right\}$ is a finite set of closed subsets of $U / Q$, there are disjoint open subsets $W_{j}$ of $U / Q$ with $K_{j} \subset W_{j}$. Now for each $j$ we define $V_{j}=W_{j} \cap U_{i}$ (for some $U_{i}$ that contains $\left.K_{j}\right)$. Note that $\left\{\alpha^{-1} V_{j}\right\}_{j=0, \ldots, k}$ is an open covering of Fix $\left(\bar{\omega}_{1}\right)$ by disjoint sets, and by 4.9 (b) $\operatorname{Ind}\left(\bar{\omega}_{1}\right)=\sum_{j=0}^{k} \operatorname{Ind}\left(\left.\bar{\omega}_{1}\right|_{\alpha^{-1} V_{j}}\right)$. Each of the open sets $\alpha^{-1} V_{j}$ is a disjoint union of $p$ open sets, each of which is homeomorphic to $V_{j}$. Let $V$ denote 
one of them, then $\alpha^{-1} V_{j}=\coprod_{q \in Q} q V$ and $\operatorname{Ind}\left(\left.\bar{\omega}_{1}\right|_{\alpha^{-1} V_{z}}\right)=\sum_{q \in Q} \operatorname{Ind}\left(\left.\bar{\omega}_{1}\right|_{q V} \dot{)}\right.$. By 4.10, $\operatorname{Ind}\left(\left.\bar{\omega}_{1}\right|_{q V}\right)=\operatorname{Ind}\left(\left.\bar{\omega}_{1}\right|_{V}\right)$ for each $q \in Q$ (note that $\bar{\omega}_{1}$ is a $Q$-equivariant map and $\left.\bar{\omega}_{1}\right|_{q V}=\left(\left.q \bar{\omega}_{1} q^{-1}\right|_{q V}\right)$.

Proof of 4.8. Note that the sheaf map $i^{*}: \mathcal{H} \rightarrow \mathcal{H}_{s}$ induced by inclusions $\hat{\pi}_{s}^{-1}(U) \rightarrow$ $\hat{\pi}^{-1}(U)(U$ open in $X / G)$ is surjective on stalks. This follows from the fact that any map from a subspace of a zero-dimensional space can be extended to entire space. Hence $i^{*}: \mathcal{H} \rightarrow \mathcal{H}_{s}$ is a quotient map. By 4.14 the restriction $\left.J(\varphi)\right|_{\operatorname{Ker}\left(i^{*}\right)}$ is 0 . Therefore $J(\varphi)$ can be factored as $\mathcal{H} \stackrel{i^{*}}{\rightarrow} \mathcal{H}_{s} \stackrel{J(\varphi)_{s}}{\rightarrow} X / G \times \mathbf{F}_{p}$. Consider the following commutative diagram:

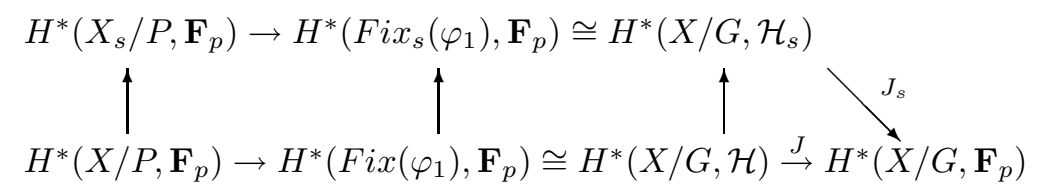

Here $J$ and $J_{s}$ are the maps induced by $J(\varphi)$ and $J(\varphi)_{s}$ respectively, and the unlabeled maps are those induced by inclusions. The bottom row composite is Oliver's transfer $\operatorname{tr} f: H^{*}\left(X / P ; \mathbf{F}_{p}\right) \rightarrow H^{*}\left(X / G ; \mathbf{F}_{p}\right)$ which factors through the top row.

\section{Relation Between two DeCompositions}

In this section we establish a relation between the $p$-toral and $p$-stubborn decompositions. The following properties of $p$-toral subgroups will be used.

Lemma 5.1 (7, 3.1, 3.2]). Let $G$ be a compact Lie group and $Q$ be a sub-p-toral subgroup of $G$.

(a) For any p-toral subgroup $P \supsetneqq Q$ in $G, N_{P}(Q) / Q$ is p-toral and nontrivial.

(b) If $G$ acts smoothly on a compact manifold $M$, then $\chi\left(M^{Q}\right) \equiv \chi(M) \bmod p$.

(c) If $N_{G}(Q) / Q$ is finite and has no nontrivial normal p-subgroup, then $Q$ is p-toral.

Lemma $5.2([\underline{6}, 1.6])$. The category $\mathcal{R}_{p}^{+}(G)$ is equivalent to a finite category.

Lemma 5.3. Let $G$ be a compact Lie group which is not a finite group of order prime to $p$. Suppose that for any p-relevant subgroup $Q$ of $G$ such that $N_{G}(Q) / Q$ is positive dimensional, $E \mathcal{O}_{p}^{+}\left(N_{G}(Q) / Q\right) \underset{p}{\stackrel{\sim}{\rightarrow}} *$. Then for any p-relevant subgroup $Q$ the fixed point space $\operatorname{ER}_{p}^{+}(G)^{Q}$ is $\bmod p$ acyclic.

Proof. To simplify the notation, denote $\operatorname{ER}_{p}^{+}(G)$ by $X$. Note that $X$ has finitely many orbit types by 5.2 Let $S$ denote the set of $p$-relevant subgroups $Q$ such that $X^{Q}$ is not $\bmod p$ acyclic. We will prove that $S$ is empty. Assuming the contrary, we order the elements of $S$ by inclusion and use Zorn's lemma to prove that $S$ has a maximal element:

Let $Q_{1} \subset Q_{2} \subset \ldots \subset Q_{i} \subset \ldots$ be a chain in $S$ and $Q$ be the closure of the union of $Q_{i}$ 's. Note that a subgroup $H$ of $G$ is sub-p-toral if and only if $(G / P)^{H} \neq \emptyset$, where $P$ is a maximal $p$-toral subgroup of $G$. For $i$ sufficiently large, $(G / P)^{Q_{i}}=(G / P)^{Q}$ and $X^{Q_{i}}=X^{Q}$ [11, Proposition IV.3.4], therefore $Q$ is in $S$. 
Now let $Q$ be a maximal element of $S$. We prove that $X^{Q}$ is $\bmod p$ acyclic and get a contradiction.

If $N_{G}(Q) / Q$ is finite and has no nontrivial normal $p$ subgroups, then $Q$ is $p$ toral and therefore $X^{Q} \simeq *$. Otherwise consider $X^{Q}$ and $(G / P)^{Q}$ as a $W=$ $N_{G}(Q) / Q$ space. The isotropy subgroups of the action of $W$ on $X^{Q}$ are of the form $\left(H \cap N_{G}(Q)\right) / Q=N_{H}(Q) / Q$ for $H$ a $p$-stubborn subgroup containing $Q$. By 5.1 (a), these subgroups of $W$ are $p$-toral and nontrivial. Let $\bar{H}$ be a nontrivial $p$-toral subgroup of $W$ and $H \subset N_{G}(Q)$ its preimage. Note that $H$ properly contains $Q$ and is a sub-p-toral subgroup of $G$ since $(G / P)^{H}$ is nonempty:

$$
\chi\left((G / P)^{H}\right)=\chi\left(\left((G / P)^{Q}\right)^{\bar{H}}\right) \equiv \chi\left((G / P)^{Q}\right) \equiv \chi(G / P) \not \equiv 0(\bmod p) .
$$

By the maximality of $Q$ the fixed point set $\left(X^{Q}\right)^{\bar{H}}=X^{H}$ is $\bmod p$ acyclic. Therefore, $X^{Q}$ is mod $p$ equivalent to $E \mathcal{O}_{p}^{+}(W)$ which is either mod $p$ acyclic by the hypothesis if $W$ is positive dimensional or contractible if $W$ has a nontrivial normal $p$-subgroup.

Theorem 5.4. Under the hypothesis of 5.3 the map $\operatorname{ER}_{p}^{+}(G) \rightarrow E \mathcal{O}_{p}^{+}(G)$ induced by the inclusion of categories $\mathcal{R}_{p}^{+}(G) \rightarrow \mathcal{O}_{p}^{+}(G)$ is a $G$-mod-p-equivalence, which implies that one of the collections is ample if and only if the other is.

Proof. The map induces mod $p$ homology isomorphism on the fixed point sets $E \mathcal{R}_{p}^{+}(G)^{H} \rightarrow E \mathcal{O}_{p}^{+}(G)^{H}$ for every isotropy subgroup $H$ of $E \mathcal{R}_{p}^{+}(G)$ or $E \mathcal{O}_{p}^{+}(G)$. The result follows from 2.6 .

Theorem 5.5. Suppose that $G$ is a compact Lie group that contains an element of order p. Under the hypothesis of 5.3, the collection of nontrivial p-stubborn subgroups of $G$ is sharp, and in particular, ample.

Proof. Let $P$ be a maximal $p$-toral subgroup of $G$. Consider the $P$ space $\left(E \mathcal{R}_{p}^{+}(G)\right)_{s}$ $=\left\{x \in E \mathcal{R}_{p}^{+}(G): G_{x} \cap P\right.$ is $p$-relevant $\}$. By definition, isotropy subgroups of $\left(E \mathcal{R}_{p}^{+}(G)\right)_{s}$ are $p$-relevant and for every isotropy subgroup $Q$ of $\left(E \mathcal{R}_{p}^{+}(G)\right)_{s}$, including $P,\left(E \mathcal{R}_{p}^{+}(G)\right)_{s}^{Q}=E \mathcal{R}_{p}^{+}(G)^{Q}$ is $\bmod p$ acyclic by 5.3 . Therefore, the projection map $\left(\operatorname{ER}_{p}^{+}(G)\right)_{s} \rightarrow *$ is a $P$-mod-p-equivalence by 2.6 The result follows from 4.3 and 4.6 .

\section{Proof of the homology DeComposition theorem}

In this section we complete our proof of 1.3 First we observe the following elementary facts:

Lemma 6.1. Suppose that $G$ is a compact Lie group and $\pi_{0}(G)$ is a p-group. If $N$ is a proper subgroup of $G$ that contains $G_{0}$, then $N$ is a proper subgroup of its normalizer in $G$.

Proof. The $p$-group $N / G_{0}$ acts on $G / N$ by left translation. The fixed point set of this action contains a nontrivial coset $g N$. The element $g$ is in the normalizer of $N$ and not in $N$.

Lemma 6.2. Let $G$ be a compact Lie group and $N$ be a normal subgroup of $G$ that contains $G_{0}$. If $P$ is a maximal p-toral subgroup of $G$, then $P \cap N$ is a maximal p-toral subgroup of $N$. 
Proof. Clearly, $P_{0}$ is the identity component of $P \cap N$ and $(P \cap N) / P_{0}$ is a subgroup of the $p$-group $P / P_{0}$, hence $P \cap N$ is $p$-toral. Since $N$ is normal in $G$, the coset space $N /(N \cap P)$ is homeomorphic to $N P / P$. Therefore, $\chi(N /(N \cap P))=\chi(N P / P) \not \equiv 0$ mod $p$ which implies the maximality of $N \cap P$ in $N$.

Lemma 6.3. Suppose that $G$ is a compact Lie group, $\pi_{0}(G)$ is a p-group, $N$ is a subgroup of $G$ that contains $G_{0}$. If $P$ is a maximal p-toral subgroup of $G$, then $P \cap N$ is a maximal p-toral subgroup of $N$.

Proof. By 6.1 there exists a normal series $N=N_{1} \triangleleft N_{2} \triangleleft \ldots \triangleleft N_{m}=G$. The proof is by downward induction on the subscript of $N_{i}$. The inductive step is provided by 6.2 .

Now we are ready to prove the main result. Note that under the hypothesis of 5.3 the conclusion of 1.3 has been proven in the previous section (5.5 and 5.4). The following theorem implies this hypothesis.

Theorem 6.4. For any positive dimensional compact Lie group $G$, the $G$-space $E \mathcal{O}_{p}^{+}(G)$ is $\bmod p$ acyclic.

Proof. The proof is by induction on the dimension of $G$.

When $\operatorname{dim} G=0$ the statement of the theorem is vacuously true.

Let $n$ be a positive integer. Suppose the theorem holds for any positive dimensional $G$ whose dimension is less than $n$. First we prove that $E \mathcal{O}_{p}^{+}(G) \underset{p}{\sim} \underset{\sim}{\sim} *$ for compact Lie group $G$ for which $\operatorname{dim} G=n$ and $\pi_{0}(G)$ is a $p$-group and then proceed to an arbitrary compact Lie group of dimension $n$.

Suppose $\pi_{0}(G)$ is a finite $p$-group. We consider two cases:

Case 1. For any $p$-relevant subgroup $Q$ of $G, \operatorname{dim} N_{G}(Q) / Q<\operatorname{dim} G$. Then by the inductive assumption the hypothesis of Lemma 5.3 is satisfied and hence by $5.5 E G \times_{G} E \mathcal{R}_{p}^{+}(G) \underset{p}{\sim} \vec{\sim} B G$. Now 5.4 implies that $E G \times_{G} E \mathcal{O}_{p}^{+}(G) \underset{p}{\sim} B G$, and an application of 3.1 shows that $E \mathcal{O}_{p}^{+}(G) \underset{p}{\stackrel{\sim}{\vec{p}}} *$.

Case 2. There is a $p$-relevant subgroup $Q$ of $G$ with $\operatorname{dim} N_{G}(Q) / Q=\operatorname{dim} G$. Then obviously $Q$ is a finite group and its normalizer $N=N_{G}(Q)$ contains the identity component of $G$. Let $K$ denote the normal subgroup of $G$ which is the intersection of all maximal $p$-toral subgroups of $G$. We will show that $K$ contains an element of order $p$.

Let $P$ be a maximal $p$-toral subgroup of $G$ that contains $Q$. The $\operatorname{subgroup~} Q$ is contained in $P \cap N$, which is a maximal $p$-toral subgroup of $N$ by 6.3. Since $Q$ is normal in $N$, it is contained in the intersection of maximal $p$-toral subgroups of $N$, which is, again by [6.3, $K \cap N$. Therefore, $K$ contains $Q$ and hence an element of order $p$.

If $G$ is itself a $p$-toral group, then $E \mathcal{O}_{p}^{+}(G) \simeq *$.

If $G$ is not $p$-toral, then for any $p$-toral subgroup $H$ of $G \operatorname{dim} H<n$ and, since $K H$ is contained in some maximal $p$-toral subgroup of $G, \operatorname{dim} K H<n$. If $K$ is positive dimensional, then by the inductive assumption $E \mathcal{O}_{p}^{+}(K H) \underset{p}{\tilde{\vec{p}}} *$ and $E \mathcal{O}_{p}^{+}(G) \underset{p}{\stackrel{\sim}{\rightarrow}} *$ by 3.5 If $K$ is finite, then the group of its inner automorphisms $A=G / C_{G}(K)$ is also finite and is a $p$-group. The action of $A$ on $K$ fixes a subgroup $K^{A}$ of $K$ and $\left|K^{A}\right| \equiv|K| \equiv 0$. The Sylow $p$-subgroup of $K^{A}$ is a nontrivial normal $p$-subgroup of $G$ and $E \mathcal{O}_{p}^{+}(G) \simeq *$ by 3.3 
This finishes the proof of the case when $\pi_{0}(G)$ is a $p$-group.

The case of an arbitrary compact Lie group of dimension $n$ now follows by an application of 3.5 .

\section{REFERENCES}

[1] A. K. Bousfield and D. M. Kan, Homotopy limits, completions and localizations, Lecture Notes in Math. 304, Springer-Verlag, Berlin, 1972. MR 51:1825

[2] A. Dold, Fixed point index and fixed point theorem for Euclidean neighborhood retracts, Topology 4, (1965), 1-8. MR 33:1850

[3] W. G. Dwyer, Homology decompositions for classifying spaces of finite groups, Topology 36 (1997), 783-804. MR 97m:55016

[4] J. Hollender and R. M. Vogt, Modules of topological spaces, applications to homotopy limits and $E_{\infty}$ structures, Arch. Math. 59 (1992), 115-129. MR 93e:55015]

[5] S. Illman, Equivariant singular homology and cohomology, Mem. Amer. Math. Soc. 156, 1975. MR 51:11482

[6] S. Jackowski, J. E. McClure and R. Oliver, Homotopy classification of self-maps of BG via $G$-actions. I, II, Annals of Math. 135 (1992), 183-270. MR 93e:55019a MR 93e:55019b

[7] S. Jackowski and R. Oliver, Vector bundles over classifying spaces of compact Lie groups, Acta Math., 176 (1996), 109-143. MR 97h:55005

[8] L. G. Lewis, J. P. May, M. Steinberger, Equivariant stable homotopy theory, Lecture Notes in Math. 1213, Springer Verlag (1986). MR 88e:55002

[9] R. Oliver, A transfer homomorphism for compact Lie group actions, Mathematische Annalen 260 (1982), 351-374. MR 83m:57034

[10] J. Slomińska, Homology decompositions of Borel constructions, preprint (Toruń) 1996.

[11] T. tom Dieck, Transformation groups. de Gruyter Stud. Math., 8. de Gruyter, Berlin-New York, 1987. MR 89c:57048

[12] S. J. Wilson, Equivariant homology theories on G-complexes, Trans. Amer. Math. Soc. 212 (1975), 155-171. MR 51:14028

Department of Mathematics, University of Notre Dame, Notre Dame, Indiana 46556

E-mail address: alexei.strounine.1@nd.edu 